\title{
The Lack of Hepatocyte Steatosis in Adult-onset Type II Citrullinemia Patients as Assessed by 7-year Interval Paired Biopsies
}

\author{
Hiroyuki Suzuki ${ }^{1}$, Yusuke Kawamura ${ }^{1}$, Keiichi Kinowaki ${ }^{2}$, Norio Akuta ${ }^{1}$, Kayoko Kasuya ${ }^{1}$, \\ Shunichiro Fujiyama ${ }^{1}$, Hitomi Sezaki ${ }^{1}$, Tetsuya Hosaka ${ }^{1}$, Satoshi Saitoh ${ }^{1}$, \\ Masahiro Kobayashi ${ }^{1}$, Mariko Kobayashi ${ }^{3}$, Yasuji Arase ${ }^{1}$, Kenji Ikeda ${ }^{1}$, Fumitaka Suzuki ${ }^{1}$, \\ Yoshiyuki Suzuki ${ }^{1}$ and Hiromitsu Kumada ${ }^{1}$
}

\begin{abstract}
:
Adult-onset type II citrullinemia (CTLN2) is a urea cycle disease characterized by neurological and psychiatric abnormalities associated with hyperammonemia. One of the pathological features of CTLN2 is the presence of hepatocyte steatosis. The condition progresses in almost all CTLN2 patients to nonalcoholic fatty liver disease (NAFLD). We herein report a 74-year-old woman who developed CTLN2 without hepatocyte steatosis. The diagnosis was based on clinical and laboratory findings and confirmed by two liver biopsies conducted within 7 years, as well as by a DNA analysis, which demonstrated mutations in the SLC25A13 gene. We describe a rare CTLN2 case without hepatocyte steatosis in an elderly woman who responded well to a low-carbohydrate diet.
\end{abstract}

Key words: adult onset type II citrullinemia, hepatocyte steatosis, fatty liver, pathology, NAFLD

(Intern Med 58: 1891-1895, 2019)

(DOI: 10.2169/internalmedicine.2374-18)

\section{Introduction}

Adult-onset type II citrullinemia (CTLN2) is a urea cycle disease characterized by acute neuropsychological manifestations associated with hyperammonemia, such as disorientation, abnormal behavior, convulsions, and death due to brain edema (1). While recent studies have highlighted the importance of nutritional support, such as low carbohydrate diet, sodium pyruvate and arginine, for improving the prognosis of CTLN2, liver transplantation remains the only definitive therapy for CTLN2 (2-6). CTLN2 is caused by a dysfunction of citrin, which is a mitochondrial aspartate-glutamate carrier, one of the urea cycle enzymes, and encoded by the SLC25A13 gene (7). Citrin dysfunction leads to a rise in the cytosolic NADH level in the liver, together with an overproduction of fatty acids and the suppression of the fatty acid metabolism (8-10). Since the carbohydrate metabolism increases the cytosolic NADH levels in the liver, carbohydrate-rich foods in CTLN2 patients could worsen the metabolic state in the hepatocytes (3). One of the clinical features of CTLN2, explained by the abovementioned pathology, is food preferences for protein- and fat-rich foods, such as peanuts, butter and cheese, and the avoidance of carbohydrate-rich foods, such as rice, sweets and alcohol (9). Although the body mass index (BMI) is $<20 \mathrm{~kg} / \mathrm{m}^{2}$ in $90 \%$ of CTLN2 patients, one of the pathological features of CTLN2 patients is the presence of a fatty liver $(10,11)$.

A genetic analysis [mutations in the SLC25A13 gene (7)] is essential in the diagnosis of CTLN2 (prepared by the research group and approved by the academic society), whereas hepatic steatosis is not necessary for a definitive diagnosis. In fact, previous studies that included liver biopsy in the diagnostic workup of CTLN2 found hepatocyte stea-

${ }^{1}$ Department of Hepatology, Toranomon Hospital, Japan, ${ }^{2}$ Department of Pathology, Toranomon Hospital, Japan and ${ }^{3}$ Department of Liver Research Laboratory, Toranomon Hospital, Japan

Received: November 12, 2018; Accepted: January 7, 2019; Advance Publication by J-STAGE: February 25, 2019

Correspondence to Dr. Norio Akuta, akuta-gi@umin.ac.jp 

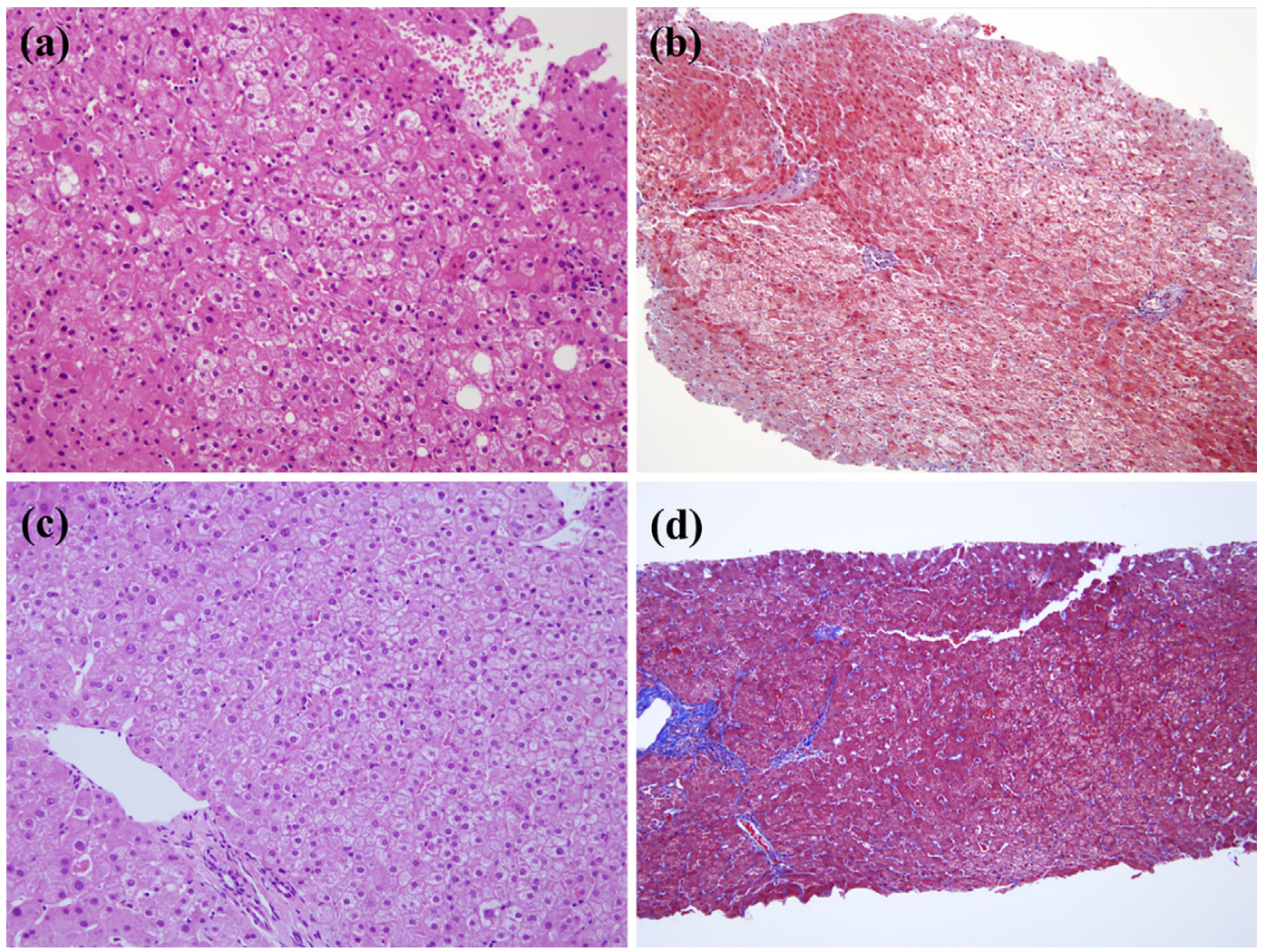

Figure 1. Histopathological findings. (a) Grade 0 steatosis (3\%) (Hematoxylin and Eosin (H\&E) staining, $\times 200$ ). (b) Grade 1 fibrosis (Masson trichrome $\times 80)$. (c) Grade 0 steatosis $(<1 \%)$. Note the absence of Mallory body and hepatocyte ballooning (H\&E staining, $\times 200)$. (d) Grade 1 fibrosis (Masson trichrome $\times 80$ ).

tosis in almost all CTLN2 patients, and these patients subsequently developed nonalcoholic fatty liver disease (NAFLD) and nonalcoholic steatohepatitis (NASH) $(5,12-15)$.

In this report, we describe a rare case of a CTLN2 patient with a histopathologically confirmed lack of hepatocyte steatosis who was assessed by 7-year interval paired biopsies and treated successfully with a low carbohydrate diet.

\section{Liver histopathology}

We obtained written informed consent from the patient before performing a ultrasonography-guided percutaneous liver biopsy and the use of genomic DNA data. Liver specimens were obtained with a 16-gauge core tissue biopsy needle (Bard Peripheral Vascular, Tempe, USA). The specimen was fixed in $10 \%$ formalin and the prepared sections were stained with Hematoxylin-Eosin (H\&E) and Masson trichrome after diastase digestion. Four pathologists (authors, $\mathrm{KK}, \mathrm{FK}, \mathrm{TF}$, and TF), who were blinded to the clinical findings, evaluated each specimen, and the final diagnosis was made by consensus. An adequate liver biopsy sample was defined as a specimen longer than $1.5 \mathrm{~cm}$ and/or containing more than 11 portal tracts. Steatosis grade 0, 1, 2, and 3, corresponded to steatosis of $<5 \%, \geq 5$ to $<33 \%, \geq 33$ to $<66 \%$, and $\geq 66 \%$, respectively. Lobular inflammation with no foci, $<2$ foci, $2-4$ foci, and $\geq 4$ foci per $200 \times$ field was scored as $0,1,2$, and 3 , respectively. Hepatocyte ballooning of none, few, and many cells was scored as 0,1 , and 2 , re- spectively. The fibrosis stage of none, zone 3 peri-sinusoidal fibrosis (stage 1), zone 3 peri-sinusoidal fibrosis with portal fibrosis (stage 2), zone 3 peri-sinusoidal fibrosis and portal fibrosis with bridging fibrosis (stage 3), and cirrhosis (stage 4) was scored as $0,1,2,3$, and 4, respectively $(16,17)$.

\section{Case Report}

A 74-year-old woman was admitted to a local hospital in May 2009 for an acute impairment of consciousness and urinary incontinence. On admission, a clinical examination showed flapping tremor and blood tests showed hyperammonemia $\left(\mathrm{NH}_{3}\right.$ : $\left.151 \mu \mathrm{g} / \mathrm{dL}\right)$, though imaging studies were negative for chronic liver disease, porto-systemic shunt, and fatty liver. The brain magnetic resonance imaging (MRI) findings were normal, and there were no clinical or imaging abnormalities that could explain the disturbance of consciousness (data not shown). She was diagnosed to have hepatic encephalopathy and was treated with oral lactulose and a low-weight protein diet. Ultrasonography-guided percutaneous liver biopsy (performed in December 2009), showed no evidence of liver cirrhosis, grade 0 steatosis (3\%), and fibrosis stage 1 (Fig. 1a and b). While her consciousness improved temporarily, the frequency of encephalopathy attacks with hyperammonemia increased. She was referred to our hospital in October 2016 for further investigation. Chronic hepatitis and fatty liver changes were ruled out based on the 

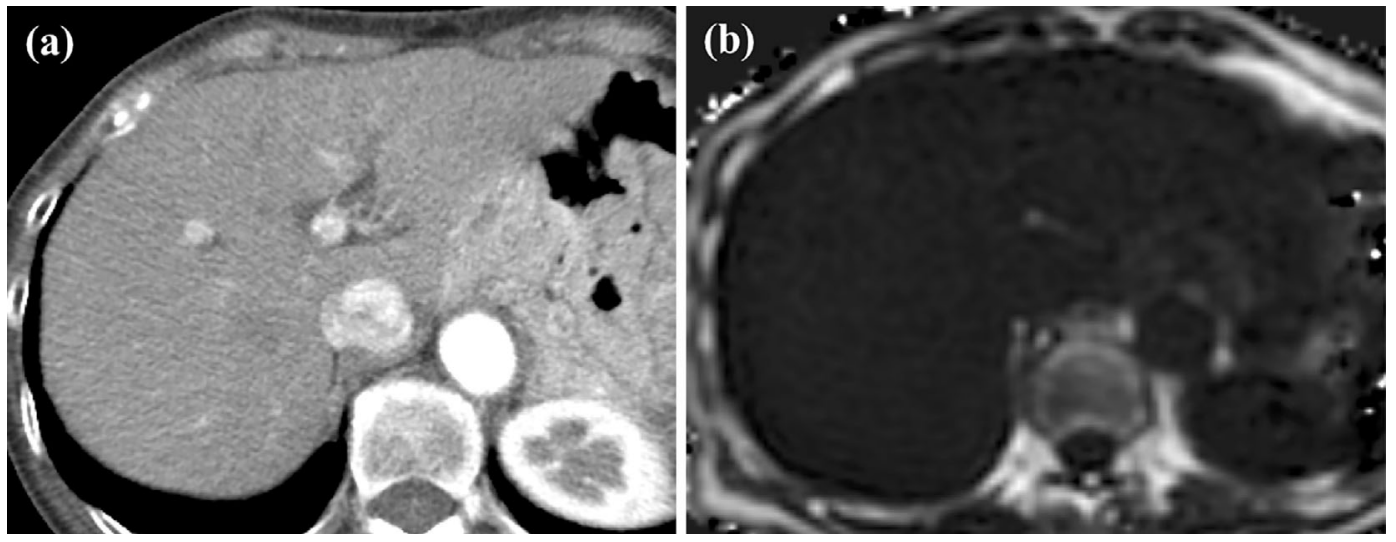

Figure 2. Imaging findings. (a) Contrast-enhanced CT shows a normal liver, (b) MRI-PDFF shows $2.4 \%$ hepatic steatosis.

Table. Clinicopathological Data.

\begin{tabular}{|c|c|c|c|c|c|}
\hline \multirow{3}{*}{$\begin{array}{c}\text { Age (years) } \\
\mathrm{Wt}(\mathrm{kg}) / \mathrm{Ht}(\mathrm{m}) / \mathrm{BMI}\left(\mathrm{kg} / \mathrm{m}^{2}\right)\end{array}$} & \multicolumn{2}{|c|}{67} & \multicolumn{3}{|c|}{74} \\
\hline & \multicolumn{2}{|c|}{ NA/1.56/NA } & \multicolumn{3}{|c|}{$40.0 / 1.56 / 16.4$} \\
\hline & on admission* & at biopsy* & on admission ${ }^{\mathbb{I}}$ & at biopsyd & after treatment ${ }^{\mathrm{II}}$ \\
\hline Ammonia $(6-51 \mu \mathrm{g} / \mathrm{dL})^{\S}$ & 151 & 64 & 132 & 34 & 39 \\
\hline Citrulline $(20-48 \mathrm{nmol} / \mathrm{mL})^{\S}$ & - & 419.5 & 865.3 & 501.8 & 336.5 \\
\hline Arginine $(70-128 \mathrm{nmol} / \mathrm{mL})^{\S}$ & - & 268.2 & 358.1 & 348.3 & 280.2 \\
\hline Threonine $(39-74 \mathrm{nmol} / \mathrm{mL})^{\S}$ & - & 108.9 & 142.8 & 129.5 & 172.3 \\
\hline Serine $(77-150 \mathrm{nmol} / \mathrm{mL})^{\S}$ & - & 50.1 & 57.1 & 47.8 & 60.1 \\
\hline Threonine-to-serine ratio & - & 2.17 & 2.5 & 2.71 & 2.87 \\
\hline AST / ALT (IU/L) & $39 / 38$ & $40 / 39$ & $23 / 16$ & $23 / 17$ & $24 / 29$ \\
\hline Albumin (g/dL) & 3.2 & 3.2 & 3.5 & 3.5 & 3.6 \\
\hline Platelet $\left(\times 10^{4} / \mu \mathrm{L}\right)$ & 32.5 & 32.5 & 25.7 & 25.4 & 25.5 \\
\hline Total cholesterol (mg/dL) & - & - & 177 & - & 168 \\
\hline Triglyceride $(\mathrm{mg} / \mathrm{dL})$ & - & - & 49 & - & 51 \\
\hline Magnetic Resonance Imaging & & NA & & Normal liver & \\
\hline Ultrasonography & & Normal liver & & Normal liver & \\
\hline Liver histopathology (date) & & 2009 & & 2016 & \\
\hline Steatosis (Grade $1 \%$ ) & & $0 / 3 \%$ & & $0 /<1 \%$ & \\
\hline Ballooning & & 2 & & 0 & \\
\hline Lobular inflammation & & 2 & & 1 & \\
\hline Fibrosis stage & & 1 & & 1 & \\
\hline Mallory Body & & + & & - & \\
\hline Glycogen nucleus & & + & & + & \\
\hline
\end{tabular}

*another hospital, ${ }^{\mathbb{I}}$ our hospital, ${ }^{\S}$ normal range

NA: not available, AST: aspartate aminotransferase, ALT: alanine aminotransferase

results of blood tests and contrast-enhanced CT and proton density fat fraction (PDFF) using MRI (18) (Fig. 2). Ultrasonography-guided percutaneous liver biopsy showed grade 0 steatosis $(<1 \%)$, and fibrosis stage 1 , but the absence of Mallory body or hepatocyte ballooning (Fig. 1c and d). She reported drinking alcohol socially only at less than $20 \mathrm{~g}$ per day, and her food preference was protein- and fat-rich foods, such as peanuts and butter since childhood. No comorbidity was found, such as diabetes or hypertension, which can be risk factors for hepatic steatosis. A serum amino acid analysis showed high levels of serum citrulline and arginine, while a DNA analysis demonstrated compound heterozygosity with [II]c.1018+1G $>$ A and [IV]
c.674C $>$ A mutations in the SLC25A13 gene (7). The final diagnosis was CTLN2 based on the above findings. A lowcarbohydrate diet (protein: fat: carbohydrate ratio= 20:40: $40 \%$ ) was started. This was followed by a decrease in the frequency of encephalopathy attacks with hyperammonemia (Table).

\section{Discussion}

CTLN2 patients show a unique dietary preference for protein- and fat-rich foods, such as beans and peanuts, and often avoid carbohydrate-rich foods, such as rice and alcohol, as a compensatory response to hepatocyte metabolic 
dysfunction. Accordingly, hepatocyte metabolic dysfunction worsens when CTLN2 patients are erroneously diagnosed as hepatic encephalopathy and placed on low-weight protein diet. This is the first case demonstrating that hepatic encephalopathy could be worsened by carbohydrate-rich food even when CTLN2 is not associated with hepatocyte steatosis. Although almost all CTLN2 cases dislike the consumption of alcohol since it rich in carbohydrates and causes fatty liver changes, many CTLN2 patients could be wrongfully misdiagnosed as NASH. Thus, even in patients with acute neuropsychological disorders associated with hyperammonemia, who have a low BMI and are negative for hepatocyte steatosis, an analysis of the dietary habits and serum amino acid levels may help in establishing the diagnosis. Unfortunately, a serum pancreatic secretory trypsin inhibitor (PSTI) gene analysis was not available at the time of the clinical management. In this regard, high serum PSTI levels have been reported to be a useful diagnostic marker for CTLN2 (19).

Saheki et al. $(1,9)$ reported that the prevalence of CTLN2 in Japan was approximately 1/100,000- 230,000 persons, while the number of male CTLN2 patients is 2.4 times higher than that of females. In this case report, although the patient had the typical dietary habit of CTLN2, no symptoms were noted until she was 67 years old without hepatocyte steatosis, thus representing a long-term survival without liver transplantation. While we did not analyze the mechanism of the latent appearance of overt clinical features of CTLN2 in our patient, it is possible that such a phenotype is more likely to be seen in females than males, based on a possible gender difference in resistance to the onset of CTLN2 and the suppression of fat deposition in the liver. In this regard, Inui et al. (20) also reported an 8-year-old girl with CTLN2 who presented with chronic hepatitis without hepatic steatosis.

Komatsu et al. (13) reported that the expression of peroxisome proliferator activated receptor $\alpha(\operatorname{PPAR} \alpha)$, one of the master regulators of the hepatic lipid metabolism, is downregulated in CTLN2 patients and inversely correlated with the severity of hepatocyte steatosis. Furthermore, four separate groups who studied 35 cases of CTLN2, including a liver biopsy, and all demonstrated the presence of mild to severe hepatocyte steatosis (12-15). To our knowledge, however, our case is the first without hepatocyte steatosis, as confirmed twice by histopathological examination based on the findings of 7-year interval paired biopsies. We cannot, however, exclude the possibility, that this heterogeneity is due to sampling variability by the liver biopsy. In this report, the histopathological findings closely correlated with those of imaging studies, such as MRI and ultrasonography. Furthermore, the findings were confirmed in the biopsies obtained 7-year apart, which both showed a lack of fatty liver changes. Accordingly, we believe that the histopathological assessment was reasonable.

In conclusion, we herein described the first known case of CTLN2 which was free of hepatocyte steatosis.

\section{Author's disclosure of potential Conflicts of Interest (COI).}

Norio Akuta: Honoraria, Bristol-Myers Squibb and Abb Vie. Yoshiyuki Suzuki: Honoraria, Bristol-Myers Squibb and Abb Vie. Hiromitsu Kumada: Honoraria, MSD, Bristol-Myers Squibb, Gilead Sciences, AbbVie and Dainippon Sumitomo Pharma.

\section{Acknowledgement}

We thank Shigeo Kure, Atsuo Kikuchi, Natsuko Ichinoi, from the Department of Pediatrics, Tohoku University School of Medicine, for the genetic analysis of SLC25A13 gene, and Dr. Takeshi Fujii, from the Department of Pathology, Toranomon Hospital, and also Drs. Fukuo Kondo and Toshio Fukusato, from the Department of Pathology, Teikyo University, for assistance in the histopathological diagnosis, and Dr. Ayako Kimura, from the Department of Gastroenterology, Tokyo Women's Medical University Medical Center East, for providing the liver biopsy samples.

\section{References}

1. Saheki T, Kobayashi K, Iijima M, et al. Pathogenesis and pathophysiology of citrin (a mitochondrial aspartate glutamate carrier) deficiency. Metab Brain Dis 17: 335-346, 2002.

2. Kogure T, Kondo Y, Kakazu E, et al. Three cases of adult-onset type II citrullinemia treated with different therapies: efficacy of sodium pyruvate and low-carbohydrate diet. Hepatol Res 44: 707712, 2014.

3. Nakamura M, Yazaki M, Kobayashi Y, et al. The characteristics of food intake in patients with type II citrullinemia. J Nutr Sci Vitaminol 57: 239-245, 2011.

4. Ikeda S, Yazaki M, Takei Y, et al. Type II (adult onset) citrullinaemia: clinical pictures and the therapeutic effect of liver transplantation. J Neurol Neurosurg Psychiatry 71: 663-670, 2001.

5. Kimura N, Kubo N, Narumi $S$, et al. Liver transplantation versus conservative treatment for adult-onset type II citrullinemia: our experience and a review of the literature. Transplant Proc 45: 34323437, 2013.

6. Yazaki M, Kinoshita M, Ogawa S, et al. A 73-year-old patient with adult-onset type II citrullinemia successfully treated by sodium pyruvate and arginine. Clin Neurol Neurosurg 115: 15421545, 2013.

7. Tabata A, Sheng JS, Ushikai M, et al. Identification of 13 novel mutations including a retrotransposal insertion in SLC25A13 gene and frequency of 30 mutations found in patients with citrin deficiency. J Hum Genet 53: 534-545, 2008.

8. Kobayashi K, Sinasac DS, Iijima M, et al. The gene mutated in adult-onset type II citrullinaemia encodes a putative mitochondrial carrier protein. Nat Genet 22: 159-163, 1999.

9. Saheki T, Inoue K, Tushima A, et al. Citrin deficiency and current treatment concepts. Mol Genet Metab 100 (Suppl 1): S59-S64, 2010.

10. Saheki T, Kobayashi K. Mitochondrial aspartate glutamate carrier (citrin) deficiency as the cause of adult-onset type II citrullinemia (CTLN2) and idiopathic neonatal hepatitis (NICCD). J Hum Genet 47: 333-341, 2002

11. Saheki T, Kobayashi K, Iijima M, et al. Adult-onset type II citrullinemia and idiopathic neonatal hepatitis caused by citrin deficiency: involvement of the aspartate glutamate carrier for urea synthesis and maintenance of the urea cycle. Mol Genet Metab 81: S20-S26, 2004.

12. Takagi $\mathrm{H}$, Hagiwara $\mathrm{S}$, Hashizume $\mathrm{H}$, et al. Adult onset type II citrullinemia as a cause of non-alcoholic steatohepatitis. J Hepatol 44: 236-239, 2006

13. Komatsu M, Kimura T, Yazaki M, et al. Steatogenesis in adultonset type II citrullinemia is associated with down-regulation of 
PPAR $\alpha$. Biochim Biophys Acta 1852: 473-481, 2015.

14. Hayasaka K, Numakura C, Toyota K, et al. Medium-chain triglyceride supplementation under a low-carbohydrate formula is a promising therapy for adult-onset type II citrullinemia. Mol Genet Metab Rep 1: 42-50, 2014.

15. Komatsu M, Yazaki M, Tanaka N, et al. Citrin deficiency as acause of chronic liver disorder mimicking non-alcoholic fatty liver disease. J Hepatol 49: 810-820, 2008.

16. Kleiner DE, Brunt EM, Van Natta M, et al. Design and validation of a histological scoring system for nonalcoholic fatty liver disease. Hepatology 41: 1313-1321, 2005.

17. Brunt EM, Janney CG, Di Bisceglie AM, et al. Nonalcoholic steatohepatitis: a proposal for grading and staging the histological lesions. Am J Gastroenterol 94: 2467-2474, 1999.
18. Yokoo T, Shiehmorteza M, Hamilton G, et al. Estimation of hepatic proton-density fat fraction by using MR imaging at $3.0 \mathrm{~T}$. Radiology 258: 749-759, 2011.

19. Kobayashi K, Horiuchi M, Saheki T. Pancreatic secretory trypsin inhibitor as a diagnostic marker for adult-onset type II citrullinemia. Hepatology 25: 1160-1165, 1997.

20. Inui A, Hashimoto T, Sogo T, Komatsu H, Saheki T, Fujisawa T. Chronic hepatitis without hepatic steatosis caused by citrin deficiency in a child. Hepatol Res 46: 357-362, 2016.

The Internal Medicine is an Open Access journal distributed under the Creative Commons Attribution-NonCommercial-NoDerivatives 4.0 International License. To view the details of this license, please visit (https://creativecommons.org/licenses/ by-nc-nd/4.0/).

(C) 2019 The Japanese Society of Internal Medicine Intern Med 58: 1891-1895, 2019 